\title{
Quantitative Estimation of 38 Illicit Psychostimulants in Blood by GC-APCI-QTOFMS with Nitrogen Chemiluminescence
} Detection Based on Three External Calibrators

\section{Mesihää, Samuel}

2020-03

Mesihää , S , Rasanen , I , Pelander , A \& Ojanperä , I 2020 , ' Quantitative Estimation of 38 Illicit Psychostimulants in Blood by GC-APCI-QTOFMS with Nitrogen Chemiluminescence Detection Based on Three External Calibrators ' , Journal of Analytical Toxicology , vol. 44 , pÿno. 2 , pp. 163172 . https://doi.org/10.1093/jat/bkz055

http://hdl.handle.net/10138/325672

https://doi.org/10.1093/jat/bkz055

unspecified

acceptedVersion

Downloaded from Helda, University of Helsinki institutional repository.

This is an electronic reprint of the original article.

This reprint may differ from the original in pagination and typographic detail.

Please cite the original version. 
Quantitative estimation of 38 illicit psychostimulants in blood by GC-APCIQTOFMS with nitrogen chemiluminescence detection based on three external calibrators

Samuel Mesihää ${ }^{1,2, *}$, Ilpo Rasanen ${ }^{2}$, Anna Pelander ${ }^{2}$, Ilkka Ojanperä ${ }^{1,2}$

${ }^{1}$ University of Helsinki, Department of Forensic Medicine, P.O. Box 40, FI-00014 University of Helsinki, Finland

${ }^{2}$ National Institute for Health and Welfare, Forensic Toxicology Unit, Helsinki, Finland

Author to whom correspondence should be addressed. Email: samuel.mesihaa@helsinki.fi 


\begin{abstract}
A method was developed for quantitative estimation of illicit psychostimulants in blood, with an emphasis on new psychoactive substances, based on gas chromatography nitrogen chemiluminescence detection coupled with atmospheric pressure chemical ionization quadrupole time-of-flight mass spectrometry (GC-NCD-APCI-QTOFMS). Quantitative estimation relied on the NCD's $N$-equimolar response to nitrogen, using amphetamine, 3,4methylenedioxymethamphetamine (MDMA) and methylenedioxypyrovalerone (MDPV) as external calibrators for prim, $s e c$ and tert-amines, respectively. After spiking with 38 stimulants at three concentration levels, the donor blood samples were submitted to liquid-liquid extraction at a basic $\mathrm{pH}$ followed by acylation with trifluoroacetic anhydride. All but three psychostimulants could be analyzed with a limit of quantification (LOQ) of $0.05 \mathrm{mg} / \mathrm{L}$. At LOQ, the between-day accuracy was $62.3-143.3 \%$ (mean $93.5 \%$, median $88.5 \%$ ) and precision $6.6-22.4 \% \mathrm{CV}$ (mean $15.8 \%$, median $16.1 \%$ ). In addition, eleven post-mortem blood samples, containing $0.08-2.4 \mathrm{mg} / \mathrm{L}$ of amphetamine $(\mathrm{n}=5)$, methamphetamine $(n=4)$ or MDMA $(n=4)$, were analyzed by the GC-NCD-APCI-QTOFMS method and the results were compared with an established electron ionization GC-MS method with appropriate calibration. The agreement between the two methods was $62.5-117.3 \%$. Regarding identification, the APCI source permitted detection of the intact precursor ion, or the respective acylation product, for all of the measured compounds. The GC-NCD-APCI-QTOFMS method developed here enables instant quantitative estimation of illicit psychostimulants in blood at reasonable accuracy, without the necessity of possessing the true reference standards for each analyte.
\end{abstract}

\title{
Keywords
}

Atmospheric pressure chemical ionization

External quantitative calibration

Gas chromatography

New psychoactive substances

Nitrogen chemiluminescence detection

Reference standards

Tentative identification

Time-of-flight mass spectrometry 


\section{Introduction}

According to the United Nations World Drug Report 2018, new psychoactive substances (NPS) continuously appear on the illicit drug market; a total of 803 NPS were reported during 2009-2017 (1). In Europe, approximately one additional NPS is reported every week to the EU Early Warning System (2). The difficulty of acquiring authentic reference standards sets a technical barrier to the identification and quantification of new drugs and metabolites. Furthermore, toxicological interpretation is difficult because reference concentrations for NPS and their metabolites in blood and urine are poorly understood.

The emergence of benchtop high-resolution mass spectrometry techniques, such as quadrupole time-of-flight mass spectrometry (QTOFMS) and Orbitrap coupled with efficient chromatographic separation, has enabled rapid tentative identification of emerging NPS. In this approach, an analysis is performed without prior knowledge of the retention time or mass spectrum by acquiring full-spectrum data in the untargeted data-independent acquisition mode (referred to as All Ions MS/MS by Agilent, bbCID by Bruker, $\mathrm{MS}^{\mathrm{E}}$ by Waters, for example). Here the collision energy is alternated in such a manner that the precursor ion is retained at the low energy mode when a soft ionization technique, such as electrospray ionization (ESI) or atmospheric pressure chemical ionization (APCI), is used. Correspondingly, the high energy mode produces structural MS/MS information via fragmentation without any preselection of ions. Data-independent acquisition allows analyzing samples in advance of acquiring the reference standards, as well as the reprocessing of historic all ions MS/MS data, without re-extracting the stored sample. This concept has been exploited in, for example, the suspect screening of NPS in drug users (3-6) and wastewater analysis (7,8), as well as in the retrospective investigation of NPS in post-mortem blood and urine $(9,10)$.

Quantification by MS techniques without actual reference standards is challenging due to compound-specific ion responses, which can lead to gross error in the estimation of the concentration $(11,12)$. The variation can be explained by differences in the sensitivity to mass spectrometric conditions, ion suppression or enhancement by the matrix compounds and by specific chemical properties that affect the ionization process (13). A simple and inexpensive way to escape the necessity of applying authentic reference standards is quantification with a universal detector, such as the flame ionization detector (FID), where any compound containing a known effective carbon number can be used as a reference standard (14). However, quantification with FID suffers from limited sensitivity and matrix interferences. The above-mentioned limitations can be circumvented with a nitrogen chemiluminescence detector (NCD) which has an equimolar and linear response to nitrogen. Furthermore, NCD offers a favorable signal-to-noise ratio as most of the drugs of abuse and NPS are nitrogenous compounds, while the biological matrix, especially blood, shows less nitrogen signals.

In our earlier studies, we have demonstrated the potential of a new analytical platform in NPS bioanalysis, consisting of gas chromatography (GC) coupled to an NCD and APCI-QTOFMS. In this configuration, the GC flow is divided between the NCD for quantification, utilizing the detector's equimolar response to nitrogen, and QTOFMS for accurate mass-based identification (15). The GC-NCD-APCI-QTOFMS was applied to estimate the concentrations of three metabolites of the common stimulant NPS $\alpha$-pyrrolidinovalerophenone ( $\alpha$-PVP) in urine samples, simulating an analysis having no authentic reference standards for the metabolites and using the parent drug instead for quantitative calibration (16). In the present study, our objective was to elaborate the analytical approach further and evaluate its performance for a broader range of illicit psychostimulants by investigating 38 drugs, with an emphasis on NPS, in spiked blood samples. Consequently, a method for quantitative estimation of these substances based on external quantitative calibration with model compounds 
was developed. Information on the method's applicability to real samples was acquired by analyzing a series of postmortem case blood samples with comparison to an established quantitative electron ionization (EI) GC-MS method.

\section{Experimental}

\section{Chemicals and samples}

Trifluoroacetic anhydride (TFAA) was from Sigma-Aldrich (Steinheim, Germany). Reference standards for drugs were from various vendors (Supplementary Table S1). Blank blood from healthy volunteers ( $\mathrm{n}=5)$ and donor blood from the Finnish Red Cross Blood Service (Helsinki, Finland) were used in the GC-NCD specificity study and in the GC-NCD accuracy and precision study, respectively. These blood samples from living persons contained $0.2 \%$ of potassium oxalate and $1 \%$ of sodium fluoride. Post-mortem femoral autopsy blank blood for the GC-NCD specificity study $(\mathrm{n}=8)$ and femoral autopsy blood from post-mortem cases $(n=11)$ known to involve amphetamine, methamphetamine and methylenedioxymethamphetamine (MDMA) were collected by the forensic pathologists of the National Institute for Health and Welfare (Helsinki, Finland) into tubes containing $1 \%$ of sodium fluoride. All the samples were stored at $4^{\circ} \mathrm{C}$ prior to analysis.

\section{Sample preparation}

Freshly prepared reference standards were dissolved in methanol to obtain a $1 \mathrm{mg} / \mathrm{ml}$ stock solution. The stock solution was diluted to 1,5 and $25 \mathrm{mg} / \mathrm{L}$ working solutions, each containing a mixture of five to eight drugs. The blood sample (1 $\mathrm{ml}$ ) was transferred into a 6-ml centrifuge tube and spiked with $50 \mu \mathrm{L}$ of the internal standard (buspirone, $5 \mathrm{mg} / \mathrm{L}$ ). Blank blood used in the validation measurements was additionally spiked with $50 \mu \mathrm{L}$ of the working solution to obtain a blood sample containing $0.05,0.25$ or $1.25 \mathrm{mg} / \mathrm{L}$ of each analyte of the drug mixture. The samples were mixed with $1 \mathrm{ml}$ of $5 \%$ ammonium hydroxide and extracted with $0.5 \mathrm{ml}$ of butyl chloride/ethyl acetate (3:1 vol/vol) in a tube rotator (40 rpm, 15 $\mathrm{min}$ ). Subsequently, $0.03 \mathrm{~g} \mathrm{NaCl}$ was added and the tubes were closed with plastic caps prior to centrifuging ( $3220 \mathrm{~g}, 10$ min). The organic phase $(200 \mu \mathrm{L})$ was transferred into another $6-\mathrm{ml}$ tube and $15 \mu \mathrm{L}$ of TFAA was added. The sample was mixed and heated at $50^{\circ} \mathrm{C}$ for 15 minutes. After cooling, $700 \mu \mathrm{L}$ of $10 \% \mathrm{NaHCO}_{3}$ was added and the solution was mixed ( $3 \mathrm{~s})$ and centrifuged $(3220 \mathrm{~g}, 10 \mathrm{~min})$. An aliquot of the organic phase ( $70 \mu \mathrm{L})$ was transferred into an autosampler vial for analysis.

\section{Analytical platform}

A 7890B Series GC System equipped with a 7693 Automatic Liquid Sampler and a split/splitless injector was coupled through a G3180B Two-Way Splitter with Makeup Gas (He) to an APCI 6540 UHD Accurate-Mass QTOF mass analyzer and a 255 Nitrogen Chemiluminescence Detector (all Agilent Technologies, Santa Clara, CA).

\section{GC analysis}

The injector liner was a Single taper Ultra Inert liner with glass wool (Agilent 5190-2293). Analytical column was a DB5MS (30 $\mathrm{m} \times 0.25 \mathrm{~mm}$ id with $0.1 \mu \mathrm{m}$ film) capillary column (Agilent Technologies). After the analytical column, the GC flow was divided between the NCD and the APCI ion source through a two-way splitter, using $0.55 \mathrm{~m} \times 0.18 \mathrm{~mm}$ and $2 \mathrm{~m} \times 0.18 \mathrm{~mm}$ uncoated deactivated fused-silica post-columns to obtain a 10:1 flow ratio, respectively. The splitter pressure was $15.8 \mathrm{psi}$ and the flow ratio was calculated using the Effluent Splitter Calculator (with Makeup) (Agilent 
Technologies). In this concurrent detection, the NCD signal arose 0.02 minutes earlier than that of QTOFMS. The GC was operated in the pulsed splitless injection mode with an equilibration time of $0.75 \mathrm{~min}$ and $50 \mathrm{~mL} / \mathrm{min}$ purge flow to split vent at $0.75 \mathrm{~min}$. A pulse pressure of $50 \mathrm{psi}$ for $0.75 \mathrm{~min}$ was applied prior to using initial head pressure of $24.9 \mathrm{psi}$. The injector port temperature was $250^{\circ} \mathrm{C}$ and the transfer line temperature $320^{\circ} \mathrm{C}$. The injection volume was $5.0 \mu \mathrm{L}$. The oven temperature was initially held at $100^{\circ} \mathrm{C}$ for $0.75 \mathrm{~min}$ and then increased by $30^{\circ} \mathrm{C}$ per min to $320^{\circ} \mathrm{C}$, which was held for $6 \mathrm{~min}$. Helium was used as carrier gas at $1 \mathrm{~mL} / \mathrm{min}$ in the constant flow mode.

\section{APCI-QTOFMS analysis}

The QTOFMS was operated in the APCI positive ionization mode, drying gas (nitrogen) flow at $5.0 \mathrm{~L} / \mathrm{min}$ and gas temperature at $365{ }^{\circ} \mathrm{C}$. The current of the corona discharge needle was $1000 \mathrm{nA}$ and capillary voltage $1000 \mathrm{~V}$. The fragmentor voltage was $140 \mathrm{~V}$ and skimmer voltage $65 \mathrm{~V}$.

Mass acquisition was performed in All Ions mode, and data were recorded over the $m / z$ range of 50-500 with an acquisition rate of 5 spectra/s. Collision energy at the low energy function was $0 \mathrm{eV}$, whereas in the high energy function $22 \mathrm{eV}$ was used. External mass calibration was carried out using the APCI tuning mix (Agilent Technologies). The ion of $m / z 257.2475$ was used for internal calibration throughout the chromatographic separation. QTOFMS was operated in $2 \mathrm{GHz}$, Extended Dynamic Range mode.

Compound identification criteria were based on the retention time $( \pm 0.02 \mathrm{~min})$, mass accuracy $( \pm 2 \mathrm{mDa})$, intensity threshold of the precursor ion (>10 000 counts) and qualifier ions (> 1000 counts) found in the in-house GC-APCIQTOFMS database. A mass increment of 95.9823 Da was added to the theoretical mass of each TFAA acylation reaction product. All data were collected with MassHunter Data Acquisition B.04.00 software (Agilent Technologies). MassHunter Profinder B.06.00 software (Agilent Technologies) was used for initial data processing and compound identification.

\section{Comparison of APCI-QTOFMS responses}

Slopes for each compound were obtained from the area of a two-point calibration model $(0.05$ and $0.25 \mathrm{mg} / \mathrm{L})$ measured on five separate days with two replicates per measurement, making in total ten measurements per compound. Subsequently, the ionization response was calculated by dividing the mean slope of each compound with the mean slope of the external calibrator (amphetamine, MDMA or MDPV). Outliers with area values beyond three standard deviations from the mean were removed from the data before constructing a slope.

\section{NCD analysis}

Pyrolysis of the analytes in the NCD was carried out at $900{ }^{\circ} \mathrm{C}$ under a hydrogen flow rate of $4 \mathrm{ml} / \mathrm{min}$ and an oxygen flow rate of $9.4 \mathrm{ml} / \mathrm{min}$. Data from the NCD was collected at $50 \mathrm{~Hz}$ over the entire course of the analysis. OpenLab CDS Chemstation GC driver A.02.05.021 was used to control the GC-NCD.

A linear regression model was constructed by measuring the external calibrators at four concentration levels (three replicates each), ranging from 0.05 to $2.50 \mathrm{mg} / \mathrm{L}$, in spiked blood. To control the quantitative analysis, three external calibration standards were chosen as follows: amphetamine for primary amines, MDMA for secondary amines and MDPV 
for tertiary amines. The peak area of the measured compounds was corrected according to the relative nitrogen content prior to applying the linear regression model.

\section{Analysis of post-mortem blood}

Eleven post-mortem femoral blood samples, previously known to contain amphetamine, methamphetamine or MDMA, were analyzed by GC-NCD-APCI-QTOFMS in duplicate, and the results were compared with those from an established GC-EI-MS method, based on selected ion monitoring and appropriate calibration with true reference standards (17).

\section{Results \& Discussion \\ Identification by GC-APCI-QTOFMS}

For a majority of the studied compounds, derivatization was necessary to prevent adsorption of prim-and sec-amines into the GC system and consequently to improve their detectability in QTOFMS (18). In our established forensic casework GC-MS method (17), heptafluorobutyric anhydride (HFBA) is used for $N$-heptafluoroacylation of amines. Here we replaced HFBA with the less bulky and more active TFAA because the derivatization of methylphenidate with HFBA was unsuccessful, likely due to steric hindrance. The derivatization efficiency was checked by searching for the extracted ion chromatograms of the underivatized precursor ions or the intermediate derivatization products. All prim- and secamines were found to be completely derivatized at all concentrations $(0.05,0.25$ and $1.25 \mathrm{mg} / \mathrm{L})$, and the expected precursor $[\mathrm{M}+\mathrm{H}]^{+}$and qualifier ions, listed in Table I, were detected for all 38 compounds.

Table I. Characteristics of studied psychostimulant drugs

These observations are in line with the previous studies on GC-APCI-QTOFMS, where soft ionization significantly promoted the detection of precursor ion, thus enabling accurate mass-based tentative identification (19-21).

\section{Accuracy and precision in GC-NCD}

Recognition of chromatographic peaks at NCD was based on their parallel detection by APCI-QTOFMS. Table II shows the accuracy and precision of GC-NCD quantification for the 38 psychostimulants in spiked healthy donor blood, based on five separate experiments at three concentration levels $(0.05,0.25$ and $1.25 \mathrm{mg} / \mathrm{L})$. The LOQ was assigned $0.05 \mathrm{mg} / \mathrm{L}$, and the mean between-day accuracy and precision at LOQ was 93.5\% (62.3-143.4\%) and 15.8\% CV (6.6-22.4\%), respectively. The values were even better at the higher concentrations. Figure 1 visualizes the detector's response at the three concentration levels.

Table II. Between-day accuracy and precision for quantitative estimation of drugs by GC-NCD using equimolar response to nitrogen and external calibration with three model compounds

Figure 1. Overlaid GC-NCD chromatograms from blood samples spiked at $0.05,0.25$, and $1.25 \mathrm{mg} / \mathrm{L}$ of 4fluoroamphetamine (4-FA), ethylcathinone, 4-methylthioamphetamine (4-MTA), $\alpha$-pyrrolidinovalerophenone ( $\alpha$-PVP), camfetamine, ethylphenidate and 2-desoxypipradrol (2-DPMP)

These results are not in every case consistent with the principles of bioanalytical method validation stating that the mean accuracy and precision should be within $\pm 15 \%$ and less than $15 \% \mathrm{CV}$ (at LOQ within $\pm 20 \%$ and less than $20 \% \mathrm{CV}$ ), 
respectively (22). However, our findings suggest that the present method for quantitative estimation of psychostimulants in blood by GC-NCD approximates the true concentration with such an accuracy $( \pm 50 \%)$ that toxicologically meaningful interpretations can be made.

As for comparison, to illustrate the problems associated with MS-based quantification in the absence of the actual reference standards, we measured the same 38 compounds under the same experimental conditions by the APCIQTOFMS. Applying external calibration with amphetamine, MDMA and MDPV, similarly to the GC-NCD experiment, resulted in a fairly consistent slope with some drugs, but with others a large difference in slopes was found, up to 2.86fold (286\% error) (Table III). Moreover, the MS response is largely unpredictable as opposed to NCD that shows a more uniform performance.

Table III. Relative ion response in GC-APCI-QTOFMS using external quantitative calibration with three model compounds

\section{Specificity of GC-NCD}

The specificity of GC-NCD analysis was established with blank blood samples from post-mortem cases $(n=8)$ and from healthy volunteers $(n=5)$. The common interfering peaks from the matrix are listed in Figure 2 . The same peaks were found in samples from both post-mortem and living persons in varying intensities. Dibutylone $(6.51 \mathrm{~min})$ and methylphenidate $(6.77 \mathrm{~min})$ partially co-eluted with an interfering matrix component and MBDB (6.48 min) was completely covered by a matrix peak, which made integration difficult especially at the lowest concentration level. In addition, the common exogenous compound caffeine $(6.57 \mathrm{~min})$ was a potential interference for camfetamine (6.62 $\mathrm{min})$. The caffeine signal $\left([\mathrm{M}+\mathrm{H}]^{+}=m / z\right.$ 195.0882) was detected in all blank blood samples by APCI-QTOFMS, but only three samples contained a detectable peak in GC-NCD.

Figure 2. Overlaid GC-NCD chromatograms from blood samples of a healthy volunteer (above) and a post-mortem case (below) with commonly observed matrix peaks listed according to their retention time values

\section{Method comparison between GC-NCD and GC-EI-MS with post-mortem cases}

Eleven post-mortem blood samples, previously known to contain amphetamine $(n=5)$, methamphetamine $(n=4)$ and MDMA ( $n=4)$ as analyzed by an established GC-EI-MS method (17), were subsequently re-analyzed by GC-NCDAPCI-QTOFMS. Table IV shows that the agreement of quantitative results between the methods was $62.5-117.3 \%$, which can be considered sufficient reproducibility in post-mortem toxicology, taking into account the fundamental differences between the methods in terms of detection and quantitative calibration.

Table IV. Comparison of quantitative results for selected drugs between GC-NCD and an established GC-EI-MS method in post-mortem blood

\section{Advantages and limitations of GC-NCD-APCI-QTOFMS}

GC-NCD-APCI-QTOFMS constitutes an efficient integrated platform for the identification and quantitative estimation of nitrogen-containing drugs, the analyst having no immediate need of the respective authentic reference standards. Our new findings strengthen the previously published results on the benefits of the GC-APCI source, enabling tentative 
identification by soft ionization (19-21). However, unequivocal identification can be accepted only after confirmation analysis based on the actual reference standards afterwards. As for quantification, GC-NCD enables better chromatographic resolution and sufficient sensitivity compared with its LC counterpart $(23,24)$, making the technique amenable to bioanalysis. The $N$-equimolar response of NCD is stable with little variation, contrary to MS techniques for which unpredictable and large differences in response may exist between compounds.

However, there are some limitations concerning the use of GC-NCD-APCI-QTOFMS in bioanalysis. Firstly, appropriate derivatization of prim- and sec-amino as well as hydroxyl groups is essential. This in turn necessitates that the spectral libraries and mass databases should include the corresponding derivatives. Secondly, quantitative estimation by GC-NCD without reference standards requires calibration by suitable model compounds to compensate for all other stages of analysis but the $\mathrm{N}$-equimolar detection. Inappropriate sample preparation and/or calibration may result in a systematic bias while precision may still remain acceptable. Our results show that the choice of calibrators for the psychostimulants was largely appropriate, but this may not be obvious with other types of drugs and metabolites. Thirdly, the specificity and sensitivity of the NCD is not comparable to MS. To improve specificity, common interferences in the NCD chromatogram should be recognized using a similar matrix prior to the actual analysis and the required methodological modifications made. The sensitivity of the NCD analysis was considered adequate for the psychostimulants in blood, but there is certainly room for improvement if low-dose drugs are involved. Lastly, quantification by NCD is limited to nitrogen-containing drugs. It has been shown that the equimolar principle was truly universal regardless of the position or number of nitrogen atoms but decreased nitrogen signal was observed in such cases where nitrogen atoms were adjacent to each other [25].

\section{Conclusions}

This is the first study applying the recently introduced GC-NCD-APCI-QTOFMS platform to the accurate mass-based tentative identification and quantitative estimation of drugs in human blood, simulating an analysis having no authentic reference standards available for the drugs and using model compounds instead for external quantitative calibration. Based on three external calibrators, we have successfully quantified 35 out of 38 illicit psychostimulants with an accuracy somewhat outside of what is encountered with methods that are calibrated using authentic reference standards but at a level that meets the requirements of meaningful toxicological interpretation. However, subsequent confirmation analysis using reference standards is necessary for professionally valid reporting. We conclude that GC-NCD-APCI-QTOFMS is among the most viable approaches for the instant estimation of stimulant NPS concentrations in blood, having very few challengers within analytical toxicology.

\section{Compliance with ethical standards}

The collection and use of the blood samples obtained from living persons was undertaken with the understanding and written consent of each subject. The analysis of drugs from the autopsy specimens was performed according to the request of judicial authorities, and the study was based on an appropriate permission.

\section{References}

1. United Nations publication, Sales No. E. 18. XI. 9. World Drug Report 2018. 
2. European Monitoring Centre for Drugs and Drug Addiction. European Drug Report 2018: Trends and Developments, Publications Office of the European Union, Luxemburg.

3. Kinyua, J., Negreira, N., Ibáñez, M., Bijlsma, L., Hernández, F., Covaci, A., et al. (2015) A data-independent acquisition workflow for qualitative screening of new psychoactive substances in biological samples. Analytical and Bioanalytical Chemistry, 407, 8773-8785.

4. Mollerup, C.B., Dalsgaard, P., Mardal, M., Linnet, K. (2017) Targeted and non-targeted drug screening in whole blood by UHPLC-TOF-MS with data-independent acquisition. Drug Testing and Analysis, 9, 1052-1061.

5. Sundström, M., Pelander, A., Angerer, V., Hutter, M., Kneisel, S., Ojanperä, I. (2013) A high-sensitivity ultra-high performance liquid chromatography/high-resolution time-of-flight mass spectrometry (UHPLC-HR-TOFMS) method for screening synthetic cannabinoids and other drugs of abuse in urine. Analytical and Bioanalytical Chemistry, 405, 84638474 .

6. Rosano, T.G., Wood, M., Ihenetu, K., Swift, T.A. (2013) Drug screening in medical examiner casework by highresolution mass spectrometry (UPLC-MSE-TOF). Journal of Analytical Toxicology, 37, 580-593.

7. Bade, R., Tscharke, B.J, White, J.M., Grant, S., Mueller, J.F, O'Brien, J., et al. (2019) LC-HRMS suspect screening to show spatial patterns of New Psychoactive Substances use in Australia. Science of the Total Environment, 650, 21812187.

8. Baz-Lomba, J.A., Reid, M.J., Thomas, K.V. (2016) Target and suspect screening of psychoactive substances in sewagebased samples by UHPLC-QTOF. Analytica Chimica Acta, 914, 81-90.

9. Partridge, E., Trobbiani, S., Stockham, P., Charlwood, C., Kostakis. C. (2018) A Case Study Involving U-47700, Diclazepam and Flubromazepam-Application of Retrospective Analysis of HRMS Data. Journal of Analytical Toxicology, 42, 655-660.

10. Noble, C., Dalsgaard, P., Stybe Johansen, S., Linnet, K. (2018) Application of a screening method for fentanyl and its analogues using UHPLC-QTOF-MS with data-independent acquisition (DIA) in MSE mode and retrospective analysis of authentic forensic blood samples. Drug Testing and Analysis, 10, 651-662.

11. Liigand, P., Liigand, J., Cuyckens, F., Vreeken, R.J., Kruve, A. (2018) Ionisation efficiencies can be predicted in complicated biological matrices: A proof of concept. Analytica Chimica Acta, 1032, 68-74.

12. Hatsis, P., Waters, N.J., Argikar, U.A. (2017) Implications for Metabolite Quantification by Mass Spectrometry in the Absence of Authentic Standards. Drug Metabolism and Disposition: The Biological Fate of Chemicals, 45, $492-496$.

13. Taylor, P.J. (2005) Matrix effects: the Achilles heel of quantitative high-performance liquid chromatographyelectrospray-tandem mass spectrometry. Clinical Biochemistry, 38, 328-334.

14. Scanlon, J.T, Willis, D.E. (1985) Calculation of flame ionization detector relative response factors using the effective carbon number concept. Journal of Chromatographic Science, 23, 333-340.

15. Ojanperä, I., Mesihää, S., Rasanen, I., Pelander, A., Ketola, R.A. (2016) Simultaneous identification and quantification of new psychoactive substances in blood by GC-APCI-QTOFMS coupled to nitrogen chemiluminescence detection without authentic reference standards. Analytical and Bioanalytical Chemistry, 408, 3395-3400.

16. Mesihää, S., Rasanen, I., Ojanperä, I. (2018) Quantitative estimation of a-PVP metabolites in urine by GC-APCIQTOFMS with nitrogen chemiluminescence detection based on parent drug calibration. Forensic Science International, 286, $12-17$.

17. Ojanperä, I., Heikman, P., Rasanen, I. (2011) Urine analysis of 3,4-methylenedioxypyrovalerone in opioid-dependent patients by gas chromatography-mass spectrometry. Therapeutic Drug Monitoring, 33, 257. 
18. Kataoka, H. (1996) Derivatization reactions for the determination of amines by gas chromatography and their applications in environmental analysis. Journal of Chromatography A, 733, 19-34.

19. Hernández, F., Ibáñez, M., Portolés, T., Cervera, M.I., Sancho, J.V., López, F.J. (2015) Advancing towards universal screening for organic pollutants in waters. Journal of Hazardous Materials, 282, 86-95.

20. Li, D., Gan, L., Bronja, A., Schmitz, O.J. (2015) Gas chromatography coupled to atmospheric pressure ionization mass spectrometry (GC-API-MS): Review. Analytica Chimica Acta, 891, 43-61.

21. Portolés, T., Sancho, J.V., Hernández, F., Newton, A., Hancock, P. (2010) Potential of atmospheric pressure chemical ionization source in GC-QTOF MS for pesticide residue analysis. Journal of Mass Spectrometry, 45, 926-936.

22. Shah, V., Midha, K., Findlay J., Hill, H., Hulse, J., McGilveray, I., et al. (2000) Bioanalytical Method ValidationA Revisit with a Decade of Progress. Pharmaceutical Research, 17, 1551-1557.

23. Ojanperä, I., Ojanperä, S., Tuominen, S. (2007) Single-calibrant quantification of drugs in plasma and whole blood by liquid chromatography-chemiluminescence nitrogen detection. Journal of Chromatography B, 856, 239-244.

24. Ojanperä, S., Rasanen, I., Sistonen, J., Pelander, A., Vuori, E., Ojanperä, I. (2007) Quantification of Drugs in Plasma Without Primary Reference Standards by Liquid Chromatography-Chemiluminescence Nitrogen Detection: Application to Tramadol Metabolite Ratios. Therapeutic Drug Monitoring, 29, 423-428.

25. Yan, X. (2006) Unique selective detectors for gas chromatography: nitrogen and sulfur chemiluminescence detectors. Journal of Separation Science, 29, 1931-1945.

\section{Supplementary data}

Supplementary material is available at Journal of Analytical Toxicology online.

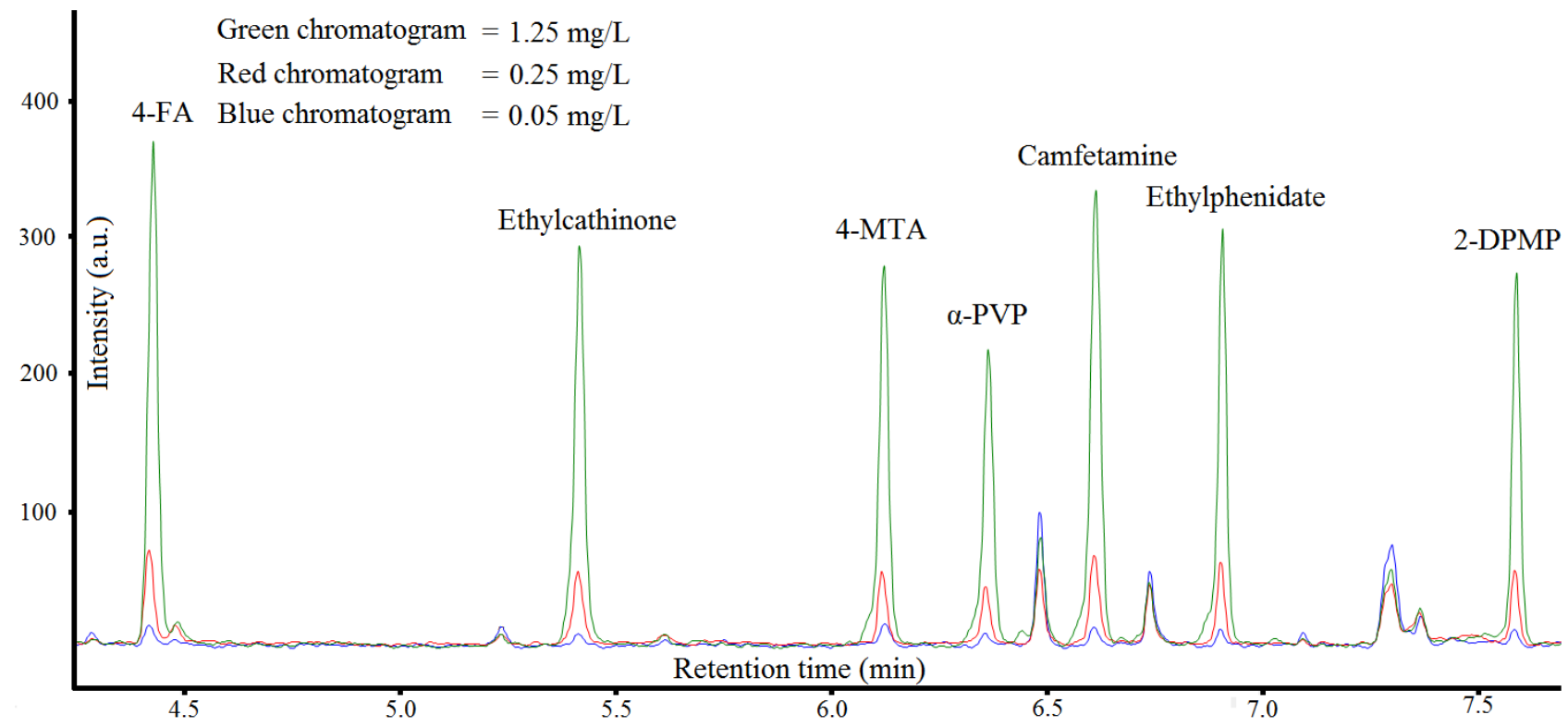




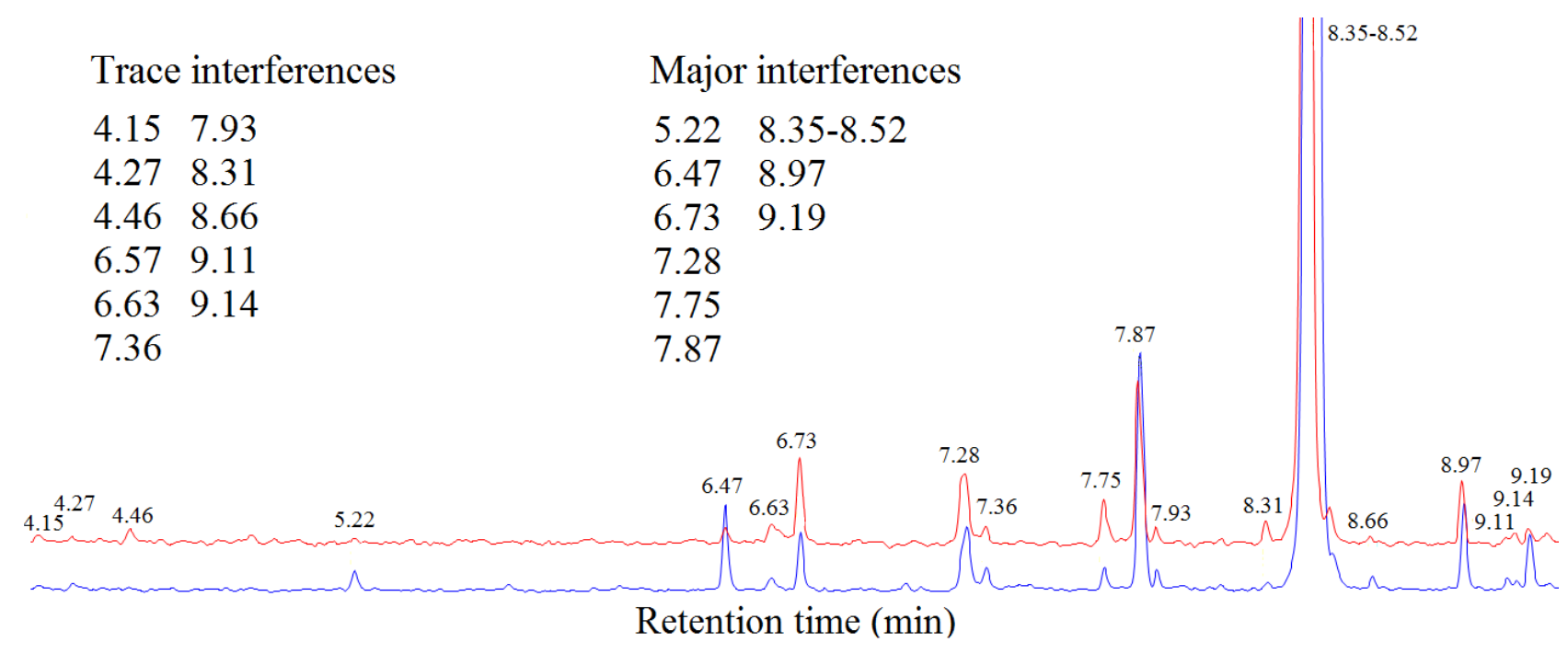


S1 Reference standard vendors for drugs used in this study

\begin{tabular}{|c|c|}
\hline Drug & Vendor \\
\hline $2 \mathrm{C}-\mathrm{B}$ & Lipomed \\
\hline 2C-T-2 & Lipomed \\
\hline $2 \mathrm{C}-\mathrm{T}-4$ & Lipomed \\
\hline 2-DPMP & Synchem \\
\hline 2-PEA & Lipomed \\
\hline 3,4-СТМР & LGC \\
\hline 3-F-Phenmetrazine & Chiron \\
\hline 4-F-Amphetamine & NMI \\
\hline 4-F-Methamphetamine & NMI \\
\hline 4-F- $\alpha-P V P$ & Chiron \\
\hline 4-Methylamphetamine & NMI \\
\hline 4-MMA & NMI \\
\hline 4-MTA & Lipomed \\
\hline 5-IT & LGC \\
\hline Amphetamine & LGC \\
\hline Amphetamine-d5 & Cerillant \\
\hline Bromo-DragonFLY & TRC \\
\hline Buspirone & Orion Pharma \\
\hline Camfetamine & LGC \\
\hline Dibutylone & LGC \\
\hline DOB & Chiron \\
\hline DOET & Lipomed \\
\hline DOM & Sigma \\
\hline Ethylamphetamine & Lipomed \\
\hline Ethylcathinone & NMI \\
\hline Ethylphenidate & LGC \\
\hline MBDB & Lipomed \\
\hline $\mathrm{m}-\mathrm{CPP}$ & NMI \\
\hline MDA & Cerillant \\
\hline MDAI & LGC \\
\hline MDEA & Lipomed \\
\hline MDMA & Chiron \\
\hline MDMA-d5 & Cerillant \\
\hline MDPV & Chiron \\
\hline Mephedrone & TRC \\
\hline Methamphetamine & LGC \\
\hline Methamphetamine-d5 & Cerillant \\
\hline Methedrone & LGC \\
\hline Methiopropamine & LGC \\
\hline Methylphenidate & Novartis \\
\hline Naphyrone & LGC \\
\hline PCP & Sigma \\
\hline Pentedrone & LGC \\
\hline Propylamphetamine & Lipomed \\
\hline$\alpha-P V P$ & Chiron \\
\hline Cerillant & Round Rock, TX, USA \\
\hline Chiron & Trondheim, Norway \\
\hline LGC & Luckenwalde, Germany \\
\hline
\end{tabular}


Lipomed

National Measurement Institute (NMI)

Novartis

Orion Pharma

Sigma

Toronto Research Chemicals (TRC)
Arlesheim, Switzerland

Pymble, Australia

Espoo, Finland

Espoo, Finland

St. Louis, MO, USA

Toronto, ON, Canada 
Primary amines

Slope relative to amphetamine

Fold change

\begin{tabular}{lll}
\hline Amphetamine & 1,00 & 1,00 \\
4-Methylamphetamine & 1,02 & 1,02 \\
DOB & 0,98 & 1,02 \\
MDAI & 0,91 & 1,10 \\
2C-T-2 & 0,82 & 1,22 \\
2-PEA & 0,81 & 1,23 \\
4-F-Amphetamine & 0,80 & 1,25 \\
DOET & 1,31 & 1,31 \\
2C-B & 0,76 & 1,32 \\
DOM & 1,36 & 1,36 \\
2C-T-4 & 0,69 & 1,45 \\
MDA & 0,67 & 1,49 \\
Bromo-DragonFLY & 0,62 & 1,61 \\
4-MTA & 0,59 & 1,69 \\
5-IT & 2,01 & 2,01
\end{tabular}

Secondary amines

Slope relative to MDMA

Fold change

\begin{tabular}{lcc}
\hline MDMA & 1,00 & 1,00 \\
3,4-CTMP & 0,95 & 1,05 \\
Methylphenidate & 1,17 & 1,17 \\
Ethylphenidate & 1,18 & 1,18 \\
Methedrone & 1,18 & 1,18 \\
4-MMA & 1,20 & 1,20 \\
MBDB & 0,83 & 1,20 \\
2-DPMP & 1,43 & 1,43 \\
Mephedrone & 1,49 & 1,49 \\
3-F-Phenmetrazine & 1,57 & 1,57 \\
Methamphetamine & 1,76 & 1,76 \\
Ethylcathinone & 1,77 & 1,77 \\
Ethylamphetamine & 1,77 & 1,77 \\
4-F-Methamphetamine & 1,80 & 1,80 \\
MDEA & 0,55 & 1,82 \\
mCPP & 1,84 & 1,84 \\
Propylamphetamine & 1,96 & 1,96 \\
Camfetamine & 1,99 & 1,99 \\
Pentedrone & 2,06 & 2,06 \\
Methiopropamine & 0,38 & 2,63 \\
Tertiary amines & & Fold change \\
\hline MDPV & Slope relative to MDPV & 1,00 \\
4-F-aPVP & 1,00 & 1,02 \\
Dibutylone & 0,98 & 1,03 \\
a-PVP & 1,03 & 1,27 \\
PCP & 0,79 & 2,78 \\
Naphyrone & 0,36 & 2,86 \\
\end{tabular}

